\title{
Child Weight and Feeding Patterns in an Old Order Mennonite Community
}

Dawn Garrett-Wright ${ }^{1}$

Assistant Professor

School of Nursing

Western Kentucky University

M. Eve Main

Associate Professor

School of Nursing

Western Kentucky University

\author{
M. Susan Jones \\ Professor \\ School of Nursing \\ Western Kentucky University
}

\begin{abstract}
Purpose: Feeding practices in some religious communities may decrease childhood obesity. However, there is limited research in these communities to assess maternal perceptions of their children's weight and feeding patterns. The purpose of this study was to ascertain Old Order Mennonite mothers' perceptions of their children's body weight and to examine feeding patterns for their children. Sample: Participants for this descriptive, correlational study were recruited from an Old Order Mennonite community in south central Kentucky. Sampling was achieved using a snowball sampling strategy and the use of a community insider. Methods: Body Mass Index was measured for mothers and child participants, and mothers completed four questionnaires. Fourteen families with 65 children participated. Findings: Fifteen children (23.1\%) had BMIs $>85 \%$, and weight was underestimated in $24.6 \%$ of children. Breastfeeding was common (98.5\%), with a mean age for cessation of 17.3 months. Mean age for introduction of solid foods was eight months. Results from the CFQ demonstrated mothers were neutral about obesity risk in their child $(\mathrm{X}=3.05)$. Mothers reported concerns about high sugar foods, limiting access to unhealthy foods, and children eating all food served at meals. Maternal health literacy scores ( $\mathrm{X}=33.98)$ were comparable to scores found in other studies. A lower PSOC score ( $\mathrm{X}=$ 23.36) was found. Conclusions: Further study needs to be conducted in this community to ascertain specific feeding habits that may account for increased BMIs.
\end{abstract}

\section{Keywords}

Hoover (Scottsville) Mennonites; Child body weight; Perceptions; Feeding patterns 


\section{Introduction}

Overweightness in children and adolescents correlates with having a Body Mass Index (BMI) between the $85^{\text {th }}$ percentile and $94^{\text {th }}$ percentile based on age and gender using the standardized growth charts (Ogden and Carroll 2012). Children with BMIs greater than the $95^{\text {th }}$ percentile are considered obese. Altogether, about $17 \%$ of American children between age two and 19 are considered obese (Ogden and Carroll 2012). This finding equates to one in every six children in our country meeting the criteria for a diagnosis of obesity. Childhood obesity is a primary concern and area for intervention due to the numerous co-morbid conditions that accompany being obese (United States Department of Health and Human Services [USDHHS] 2013a). Addressing the obesity epidemic is at the forefront of the Healthy People 2020 initiative and is listed as one of the ten leading health indicators (USDHHS 2013b). Two specific goals included in the Nutrition and Weight Status (NWS) category of Health People 2020 are NSW 10 and NSW 11, which recommend reducing the number of children and adolescents who met the criteria for obesity and to prevent inappropriate weight gain in children and adolescents (USDHHS 2013b).

Interventions to prevent and reduce childhood and adolescent obesity must focus on modifiable risk factors including dietary intake and child feeding practices in the home (SpruijtMetz, et al. 2002). These interventions may be difficult to implement given that previous research has shown that parents are often unaware of what constitutes a healthy body weight for their child and developing weight problems (Garrett-Wright 2011). Numerous studies have been done on children in modern, westernized countries, indicating increased calorie consumption and various feeding patterns are contributing factors to the rising rate of child and adolescent obesity; however, few studies have been conducted in other cultures to examine how feeding patterns affect body weight in children and adolescents.

Old Order Amish (OOA) and Old Order Mennonite (OOM) communities provide a population to assess different lifestyles compared to those in our modern, western culture. These religious groups are known as Anabaptists and draw their origins from the Anabaptist movement of the sixteenth century (Kraybill 2010). Both OOA and OOM communities adopt conservative lifestyles that typically involve agrarian culture, avoidance of many modern technologies, and varying degrees isolation from mainstream culture (Kraybill 2001; Tremblay, et al. 2008). In terms of healthcare, members of most OOM communities do not participate in private or governmental insurance programs and the use of screenings and preventative care is limited (Jones, Main, and Garrett-Wright 2013).

There are several significant lifestyle differences noted between children in OOA and OOM communities when compared with children raised in a typical Western society. Research with special populations such as OOA and OOM can serve as a natural comparison for children in traditional Western societies that have high levels of physical inactivity and a less cohesive family structure. Children in Anabaptist communities do not participate in the typical leisure 
activities of Western society (e.g. video games, watching TV) but instead spend time in active play and completing chores (Tremblay, et al. 2008). This increased activity level is hypothesized to decrease the rate of obesity in children. Some authors suggest that "lifestyle-embedded physical activity" may be more important than engaging in organized sports activities (Tremblay, et al. 2008, 837). Family meals, inherent in the OOM community, have been suggested as a protective factor against childhood weight problems (Berge, et al. 2014). Other psychosocial variables including confidence in parenting skills and health literacy have been linked to parents' recognition and understanding of increased levels of BMI (Garrett-Wright 2011) but these variables have not been studied in Anabaptist communities. The purpose of the current study was two-fold: (1) to describe the BMI of children and the feeding patterns used in an OOM community, and (2) to determine the relationships between select maternal psychosocial variables with accuracy of perception and concern about child body weight.

\section{Methods}

\section{Participants and Inclusion Criteria}

Participants in the study were mothers and children from an OOM community in south central Kentucky. Access to the Mennonite community was obtained by working with a community insider who had knowledge of the health practices and connections to the leaders within the community. Parents were recruited into the study using a snowball sampling technique. Inclusion criteria for the study included being a mother of a child two to 18 years of age, the ability to read and speak English, having a child with no chronic illness, and being a member of the selected OOM community. The study was approved by the Western Kentucky University Human Subjects Review Board as well as the bishop and the male leaders in the community. Informed consent was obtained from the mothers and assent was obtained from child participants over the age of seven years. The sample consisted of mothers and children from 14 families in the community with a total of 65 child participants.

\section{Procedures}

The primary researcher and co-investigators were informed of interested participants by a community member. The researchers travelled to the community and met with families in their homes or at the home of a community member where monthly health clinics are held. After consenting to participate, mothers and children were weighed and their height was measured using a standardized measuring tape and a calibrated portable scale. Mothers were then provided copies of four questionnaires to complete for each child. Questionnaires included a demographic questionnaire, the Parental Sense of Competence Scale (PSOC), the Test of Functional Health Literacy in Adults (Short Form) (S-ToFHLA), and the Child Feeding Questionnaire (CFQ). The mothers in the community asked to be given time to review their children's records (baby books) while completing their questionnaires; therefore, the mothers were given postage paid envelopes to return their surveys to the primary researcher. 


\section{Instruments}

\section{Demographic questionnaire}

A researcher-developed demographic questionnaire was used to capture data related to the sample characteristics and basic feeding issues. Specific demographic questions included maternal age, child age and gender, number of children living in the home, race, and highest level of education obtained. Questions related to feeding included whether or not and for how long the child breastfed, use of commercial formula or other breast milk substitutes, and age when child began eating solid foods

\section{PSOC}

The PSOC was developed originally by Gibaud-Wallston (1977) as a measure of a parent's confidence and esteem for themselves in the parenting role. Further statistical analysis of the PSOC by Johnston and Mash (1989) revealed the original scale to have two stable subscales which were labeled the Satisfaction subscale and the Efficacy subscale. For the current study, the Efficacy subscale was utilized to measure the mothers' sense of their own "competence, problem solving ability, and capability in the parenting role” (Johnston and Mash 1989, 167). There are eight questions on the PSOC-Efficacy Subscale that are answered in Likert format from 1 (strongly agree) to 6 (strongly disagree). The total score is calculated after reverse scoring the items with higher total scores indicating a greater parental confidence and self-esteem. Internal consistency values of .67 to .88 were calculated for the Efficacy Subscale in previous studies (Coleman and Karraker 2003; Johnston and Mash 1989; Lovejoy, Verda, and Hayes 1997; Ohan, Leung, and Johnson 2000; Sanders and Woolley 2005). The internal consistency for the current study was .76.

\section{S-ToFHLA}

The S-ToFHLA is a measure of a participant's ability to read and comprehend the meaning of basic healthcare information such as “pill bottles and appointment slips” (Baker, et al., 1999, 34). The S-ToFHLA consists of 36 multiple choice items, with each item being valued at 0 (incorrect) or 1 (correct). Baker, et al. (1999) suggested that scores be broken down into three possible literacy categories: Adequate (scores above 23); Marginal (scores between 17 and 22); and Inadequate (scores of 16 or less).

\section{CFQ}

The CFQ is an instrument that examines feeding patterns in children by looking at seven feeding behaviors/issues such as concern over child body weight, control issues related to feeding, restriction practices related to feeding the child, and concern about child's eating habits. Answers for each subscale are answered on a five-point Likert scale. For the purpose of this study, participants answered all seven subscales, and basic descriptive statistics were calculated 
for each subscale. Previous study has indicated internal consistency scores of .70 to .92 for the CFQ subscales (Birch, et al. 2001). The Cronbach alpha score for the full instrument for this study was .78. The Perceived Child Weight subscale has an alpha score of .80, the Food Restriction Subscale had an alpha score of .87, and the Concern about Child Overweight scale had an alpha score of .55.

\section{Accuracy of Perception}

To ascertain accuracy of perception of child body weight, the researchers compared the mothers' responses to the CFQ question about perception of their children's current weight to their child's actual current weight status (BMI). The comparison was made using the child's BMI based on Centers for Disease Control child BMI charts to determine if mothers had accurate or inaccurate perceptions. The mothers' perception was defined as "accurate" if it met one of the following criteria: (1) the child's BMI was between $5^{\text {th }}$ and $84^{\text {th }}$ percentile on CDC growth charts and the mother identified the child as having an appropriate body weight, (2) the child's BMI was $<5^{\text {th }}$ percentile on CDC growth charts and the mother identified the child as being underweight, or (3) the child's BMI was $\geq 85^{\text {th }}$ percentile on CDC growth charts and they identified the child as overweight. All other responses were labeled as “inaccurate” perceptions for the purpose of data analysis.

\section{Statistical Analysis}

Statistical analyses for the current study were completed using SPSS version 19.0. Descriptive analyses were used to summarize the findings from the demographic questionnaire, to describe the sample characteristics, and to summarize results from the PSOC, S-TOFLA, and CFQ for the sample. Pearson and Kendal Tau correlations were used to determine relationships between health literacy, parental sense of competence, subscales on the CFQ related to feeding patterns/habits, and demographic data.

\section{Results}

\section{Sample Demographics}

Fourteen mothers and 65 children participated in the study. Maternal age ranged from 26 to 47 years with a mean of 39.2 years. All mothers in the study were Caucasian and had less than a formal high school education, which was expected for this community. The mean maternal BMI for the study was 27.3 with a range from 19.4 to 48.4 .

Children in the study ranged from two to 17 years with a mean age of 8.3 years. There were 40 female children in the study (61.5\%) and 25 male children (38.5\%). All children in the study were Caucasian (100\%). In addition, almost all children in the study had been breastfed at some point in the first year of life $(n=64)$, and only six children $(9.2 \%)$ had been given commercial formula during the first year of life. Age at cessation of breastfeeding ranged from 
seven to 24 months with a mean age of 17.3 months for ending breastfeeding. The majority of children ( $n=55,84.6 \%$ ) breastfed for at least 12 months. For those using, formula the age commercial formula use ended ranged from three to 23 months ( $\mathrm{X}=10.2$ months). Children in the study began eating solids between the ages of three to 18 months with a mean age of solid food introduction of 7.9 months.

Child BMIs ranged from 12.9 to 29.3 with the mean BMI of the child participants being 17.0. A small percentage of children were underweight based on CDC BMI categories with BMIs less than $5^{\text {th }}$ percentile for age and gender $(n=4 ; 6.2 \%)$. The majority of child participants had BMIs in the normal range between the $5^{\text {th }}$ and $84^{\text {th }}$ percentile for age and gender $(n=46 ; 70.8 \%)$. There were 15 children (23.1\%) with BMIs greater than the $85^{\text {th }}$ percentile which indicates an overweight or obese status. Ten of these children had BMIs between the $85^{\text {th }}$ and $94^{\text {th }}$ percentile and were identified as overweight, with an additional five children having BMIs greater than the $95^{\text {th }}$ percentile indicating obesity.

\section{Accuracy of Parental Perceptions}

Perception of child body weight was assessed as correct or incorrect based on the mothers' identification of the children's body weight in comparison with the CDC BMI categories for body weight in children. In the current study, only $69.2 \%(n=45)$ of mothers correctly identified the weight category that matched their child's BMI. Nearly $31 \%(n=20)$ of mothers had an incorrect perception of their child's current body weight. Of those mothers who misperceived their child's body weight $(n=20)$, the majority underestimated the child's BMI $(n=16 ; 80 \%)$. Overestimation of child body mass occurred in only a small number of mothers $(\mathrm{n}=4)$ with only four children identified as being heavier than they actually were based on measurements of height and weight and subsequent BMI calculation.

\section{PSOC Results}

PSOC Efficacy subscale scores ranged from two to 32 in the sample. The mean PSOC Efficacy Subscale score was 23.4. These scores were fairly low given that PSOC Efficacy Subscale scores can range from zero to 48, with higher scores indicating a greater sense of esteem and confidence in parenting skills. The lower levels of parenting self-esteem and confidence are fitting with the Old Order Mennonite culture, which emphasizes reliance on God and community rather than self.

\section{S-ToFHLA Results}

Scores for mothers completing the S-ToFHLA instrument ranged from 19 to 36, with a mean score of 33.9. Thirteen mothers scored above 23 on the S-ToFHLA, indicating adequate ability to understand and utilize data presented in healthcare settings. The remaining mother scored in the marginal category because she did not feel comfortable completing all questions on the instrument. 


\section{CFQ Results}

The CFQ contains several sections. The first section addresses who is responsible for feeding the child, selecting portion sizes, and making sure the child eats the right kind of foods (Birch, et al. 2001). Mothers in the study had a mean score of 4.1 (with 1 being the lowest and 5 the highest score for level of responsibility), emphasizing the strong maternal role in food preparation and child feeding in this particular community. All mothers in the study reported that either most of the time or always, the responsibility of feeding the child was theirs.

Another section of the CFQ asks about parental concerns about child body weight. Questions in this section focus on concerns the child eating too much when the parent is not with them and concerns about the child becoming overweight and having to diet later in life (Birch, et al. 2001). Mennonite mothers in the sample had a mean score of 2.9 out of a possible 5 , which correlates to a neutral level of concern about these issues. For $52 \%$ of the children $(n=34)$, the mother identified being unconcerned or neutral about children eating too much when they were not with them. Mothers were unconcerned about the need for dieting in $81 \%$ of the children in the sample.

The food restriction section of the CFQ poses questions related to restricting items such as high fat foods, sweets, and favorite foods. It also addresses the use of food as a reward for good behavior (i.e. I offer my child his or her favorite foods in exchange for good behavior) (Birch, et al. 2001). The mean score for this section was 3.8 on a 1 to 5 scale, indicating that mothers did restrict certain foods and use foods as rewards in some cases. Sixty-three percent of children $(n=41)$ were reported as having some food kept out of their reach. Over a quarter of children in the study ( $\mathrm{n}=18,27.7 \%)$ received "sweets" as a reward for good behavior, and over one-third ( $n=23$; 35.4\%) were given their “favorite foods” in exchange for good behavior.

Monitoring of intake of high fat food, snacks, and sweets is also assessed on the CFQ (Birch, et al. 2001). Many children in the study were monitored by mothers for intake of high fat foods ( $n=31 ; 47.7 \%)$. Snack foods, such as chips and sweets, were monitored for 55 children (84.6\%). The mean score for mothers on this section of the questionnaire was 3.7 out of a possible 5, indicating most children were monitored some to all of the time when consuming foods that were considered to be high fat, snacks, or sweets such as candy and ice cream.

\section{Bivariate Correlations}

Several interesting relationships were noted by calculating bivariate correlations. As expected, child BMI was inversely correlated with age that breastfeeding stopped, with children with higher BMI having breastfed for shorter periods $(r=-.316, \mathrm{p}<.05)$. The age solid foods were begun was negatively correlated with restriction $(r=-.328, \mathrm{p}<.01)$ and monitoring of intake of snacks, sweets, and high fat food $(\mathrm{r}=-.273, \mathrm{p}<.05)$ for children based on responses in those sections of the CFQ. Higher health literacy was positively correlated with higher levels of concern about future weight problems and monitoring of child intake $(r=.382, \mathrm{p}<.01$ and 
$\mathrm{r}=.233, \mathrm{p}<.05$, respectively). There was a positive correlation between a mother's PSOC Efficacy subscale score and her concern for child body weight $(\mathrm{r}=.377, \mathrm{p}<.01)$, her restriction of certain foods ( $\mathrm{r}=.417, \mathrm{p}<.01)$, and her monitoring of foods $(\mathrm{r}=.267, \mathrm{p}<.05)$. There was a positive correlation between a mother reporting concern about child body and their having more restrictions on child eating habits as would be expected $(\mathrm{r}=.329, \mathrm{p}<.01)$. Mothers with higher BMIs showed less concern for future child obesity potential $(r=-.387, \mathrm{p}<.01)$. In mothers who used food as a reward or restricted access to food, there was a positive correlation with monitoring intake of certain foods (high fat, snacks, sweets) $(r=.413, \mathrm{p}<.01$ ).

\section{Discussion}

One purpose of this study was to describe the BMI in Old Order Mennonite children. In this sample, the percentage of obese children was lower than the U.S. national prevalence. Only $8 \%$ of the OOM children were identified as obese as compared to a national prevalence of $17 \%$ (Fryar, Carroll, and Ogden 2014). The percentage of overweight children more closely mirrored the U.S. prevalence as $15 \%$ of OOM children were identified as overweight as compared to a U.S. rate of 14.9\% (Fryar, Carroll, and Ogden 2014). A majority of the OOM children (70.8\%) had what is considered to be a normal BMI. The most recent U.S. national statistics indicate that $65.2 \%$ of U.S. children have a normal BMI (USDHHS 2012).

Another purpose of this study was to describe the feeding practices in this OOM community. There were many positive feeding habits noted in this sample that may contribute to healthy BMI levels in children. These include the use of breastfeeding as the primary food supply for the first year of life and delaying the onset of introducing solid foods past six months of life. According to the American Academy of Pediatrics (AAP) (2012), breastfeeding is recommended as the only source of nutrition until the age of six months and should continue until at least one year of age. In the sample, children on average met and exceeded these recommendations with the mean age of weaning at 17.3 months and solids begun on average after about eight months of age. According to the Centers for Disease Control (CDC) (2012), only about $25 \%$ of U.S. children are breastfed until 12 months of age and less than $20 \%$ receive only breast milk until age six months. Despite the positive practices related to breastfeeding and initiation of solid foods later in infancy, several children in the sample were found to have a BMI above the recommended $85^{\text {th }}$ percentile for age and gender.

The mothers in this population indicated a lower parental confidence and self-esteem compared to other populations. In a previous study in a rural Kentucky sampling of nonMennonite parents, the mean PSOC Efficacy subscale score was found to be 38.9 compared to 23.4 in the Mennonite mothers sampled in this study (Garrett-Wright 2011). This is a culturally congruent finding given that Mennonite parents heavily rely on God's will in parenting and view success with children as a blessing from God, not something they achieve by their own work (Kraybill and Bowman 2001). 
Parenting efficacy was positively correlated in the current study with concern about future obesity for the child and restriction of and monitoring of foods. This is an interesting finding given the cultural norm of relying on God to control the outcome of situations, even those related to health and wellness.

Health literacy was good in this sample of mothers, and is comparable to levels found in other rural Kentucky populations (Garrett-Wright 2011). This indicates that mothers in the sample are able to take in and understand healthcare information related to their children. This finding is noteworthy as the mothers in the OOM population are not exposed to health messages via any electronic media and the majority only attends school through the eighth grade. Additionally, OOM children are not seen in traditional healthcare settings for well child care and immunizations, but their mothers intuitively follow feeding guidelines (e.g. prolonging breastfeeding, solid food initiation, limited snacks, and high fat foods) that are discussed by healthcare providers with parents at such visits. Higher health literacy scores were also positively correlated with increased levels of concern regarding child body weight and monitoring behaviors.

\section{Limitations}

Limitations in regards to sampling, design, and measurement affected the study. Because of the nature of the population studied, the overall sample was small and homogenous. It would be beneficial to replicate this study in other English and plain Anabaptist communities to compare findings from more diverse groups. In addition, sampling was conducted via a snowball strategy in a community that is segregated in many ways from the mainstream English culture. It is possible that mothers and families who agreed to participate may have had different perceptions or feelings about body weight in children than those who did not participate, as well as about the research process in general. Another limitation is that participants in this study were all mothers from one cultural cohort; therefore, the findings cannot be generalized to parents from other geographic regions or cultures. In addition, the design of the current study is a limitation. The current study was a descriptive cross-sectional design, so maternal perceptions and child body weight were assessed at only one point in time, and causality should not be inferred. Measurement could also be considered a limitation in this sample. The researchers collected data in the participants' homes, so movement of equipment from place to place could have affected its accuracy. In addition, the questionnaires in this study were self-report and social desirability factor is a problem encountered when collecting data via self-report. Also, because the PSOC has not been used in other studies with Mennonite parents, it is possible that the tool should be analyzed for cultural biases and applicability in this culture.

\section{Conclusions}

Positive lifestyle habits related to feeding in the Mennonite community may be related to a lower level of obesity in this population as compared to other westernized populations. 
Children in this culture avoid many of the variables that are linked to obesity in Western cultures, such as excessive media use (TV and video games), limited physical activity, and a high fat, processed diet. However, overweight and obesity still affected some children in this community. Fifteen children from the sample met the criteria for overweight and/or obesity (23.1\%) and one third of children's weights were misidentified with the majority having their weight underestimated by their mothers. Elevated BMIs could put these children at risk for the serious health consequences of obesity if they are not identified early and treated. Given that seeking healthcare for preventative services is rare in this community, these children may face greater risks for health problems as they grow into adulthood. Because of the risk of obesity, the authors recommend that nurses and other health professionals form partnerships with OOM communities so basic screening and education can be provided in a culturally appropriate manner. Future longitudinal research is needed to ascertain when overweight and obesity begin to develop in this population and why the protective benefits of early positive infant feeding patterns do not persist into later childhood. In summary, future research is needed to examine how embedded activity, feeding patterns, and other lifestyle factors impact the development of potential weight problems in OOM children. Longitudinal research should examine if these childhood weight problems lead to increased negative health outcomes (e.g. hypertension, diabetes).

\section{References}

American Academy of Pediatrics. 2012. Where We Stand: Breastfeeding. (HealthyChildren.org).

Baker, David, Mark Williams, Ruth Parker, Julie Gazmararian, and Joanne Nurss. 1999. “Development of a Brief Test to Measure Functional Health Literacy.” Patient Education and Counseling 38(1):33-42

Berge, J., M. Wall, T. Hsueh, J. Fulkerson, J. Larson, and D. Neumark-Sztainer. 2014. “The Protective Role of Family Meals for Youth Obesity: 10-Year Longitudinal Associations.” The Journal of Pediatrics 165(4):DOI: 10.1016/j.jpeds.2014.08.030.

Birch, L., J. Fisher, K. Grimm-Thomas, C. Markey, R. Sawyer, and S. Johnson. 2001. "Confirmatory Factor Analysis of the Child Feeding Questionnaire: A Measure of Parental Attitudes, Beliefs, and Practices about Child Feeding and Obesity Proneness.” Appetite 36(3):201-10.

Centers for Disease Control. 2012. Breastfeeding among U.S. Children Born 2000-2009, CDC National Immunization Survey. (http://www.cdc.gov/breastfeeding/data/NIS_data/).

Coleman, Priscilla, and Katherine Hildebrandt Karraker. 2003. "Maternal Self-Efficacy Beliefs, Competence in Parenting, and Toddlers' Behavior and Developmental Status.” Infant Mental Health Journal 24(2):126-48. 
Fryar, C., M. Carroll, and C. Ogden. 2014. Prevalence of Overweight and Obesity among Children Adolescents: United States, 1963-65 through 2011-2012. (http://www.cdc.gov/nchs/data/hestat/obesity_child_11_12/obesity_child_11_12.pdf)

Garrett-Wright, Dawn. 2011. "Parental Perception of Preschool Child Body Weight.” Journal of Pediatric Nursing 2(5):435-45.

Gibaud-Wallston, J. 1977. Self-Esteem and Situational Stress: Factors Related to Sense of Competence in New Parents. Doctoral dissertation in Psychology. Nashville, TN: George Peabody College for Teachers.

Jones, M.S., M.E. Main, and D. Garrett-Wright. 2013. "Collaborative Educational Innovation in an Old Order Mennonite Community.” Journal of the National AHEC Organization, 29(1):24-27.

Johnston, Charlotte, and Eric Mash. 1989. “A Measure of Parenting Satisfaction and Efficacy.” Journal of Clinical Child Psychology 18(2):167-75.

Kraybill, Donald. 2010. Concise Encyclopedia of Amish, Brethren, Hutterites, and Mennonites. Baltimore, MD: Johns Hopkins University Press.

Kraybill, Donald, and Carl Bowman. 2001. On the Backroad to Heaven: Old Order, Hutterites, Mennonites, Amish, and Brethren. Baltimore, MD: Johns Hopkins University Press.

Lovejoy, M. Christine, Michele Verda, and Cynthia Hays. 1997. “Convergent and Discriminant Validity of Measures of Parenting Efficacy and Control.” Journal of Clinical Child Psychology 26(4):366-76.

Ogden, Cynthia, Margaret Carroll, Brian Kit, and Katherine Flegal. 2012. "Prevalence of Obesity and Trends in Body Mass Index among US Children and Adolescents, 19992010.” Jama 307(5):483-90.

Ohan, Jeneva, Debbie Leung, and Charlotte Johnston. 2000. “The Parenting Sense of Competence Scale: Evidence of a Stable Factor Structure and Validity.” Canadian Journal of Behavioural Science 32(4):251-61.

Sanders. M.R., and M.L. Woolley. 2005. “The Relationship between Maternal Self-Efficacy and Parenting Practices: Implications for Parent Training.” Child: Care, Health and Development 31(1):65-73.

Spruijt-Metz, Donna, Christin Lindquist, Leann Birch, Jennifer Fisher, and Michael Goran. 2002. "Relation between Mothers’ Child-Feeding Practices and Children’s Adiposity." American Journal of Clinical Nutrition 75(3):581-86. 
Tremblay, Mark, Dale Esliger, Jennifer Copeland, Joel Barnes, and David Bassett. 2008. "Moving Forward by Looking Back: Lessons Learned from Long-Lost Lifestyles." Applied Physiology, Nutrition, and Metabolism 33(4):836-42.

United States Department of Health and Human Services, Healthy People 2020. 2013.

Nutrition, Physical Activity, and Obesity. (http://www.healthypeople.gov/ 2020/LHI/nutrition.aspx).

United States Department of Health and Human Services, Healthy People 2020. 2013. Nutrition and Weight Status. (http://www.healthypeople.gov/2020/topicsobjectives 2020/objectiveslist.aspx?topicId=29\#144171).

United States Department of Health and Human Services, Health System Measurement Project. 2012. Percentage of Children with a Healthy Weight. (https://healthmeasures.aspe.hhs. gov/measure/36a). 